Gut, 1981, 22, 475-480

\title{
Corticosteroid treatment increases parasite numbers in murine giardiasis
}

\author{
K V NAIR,* J GILLON, AND A FERGUSON $\dagger$ \\ From the Gastro-Intestinal Unit, Western General Hospital and University of Edinburgh, Edinburgh
}

SUMMARY Corticosteroid therapy is known to be hazardous in patients with occult infection but the mechanism by which the host parasite relationship is altered by steroids is not known. We have used an intestinal protozoal parasite, Giardia muris, to examine the effects of corticosteroids on the number of parasites in the intestine in the course of a primary infection. A single injection of cortisone acetate, subcutaneously, one day before oral inoculation of CBA mice with 1000 cysts of Giardia muris, resulted in significantly higher trophozoite counts in animals studied at one, two, three, four, and eight weeks post-infection, when they were compared with saline injected controls. Recrudescence of occult infection was also achieved by cortisone acetate treatment of mice which had been infected with Giardia muris eight months previously. Clinical studies are required to establish if recrudescence of occult protozoal infection is an important cause of morbidity when immunosuppressive therapy is given to patients in areas where giardiasis is endemic.

Giardia lamblia and Entamoeba histolytica are among the few protozoan parasites of the human intestine. The effects of infection with either of these vary from the asymptomatic carrier state to severe gastrointestinal disease-a malabsorption syndrome in giardiasis, colitis in amoebiasis. It is likely that several host-related factors (age, immunity) and parasite properties (strain, virulence, number) interplay in an individual patient and there is evidence, in amoebiasis, that immunosuppression, such as that produced by corticosteroids, rapidly alters the stable host parasite relationship in the carrier and leads to acute amoebic dysentery and fulminating hepatic amoebiasis. ${ }^{1}$ It is not known how steroids, and other host factors such as pregnancy ${ }^{2}$ produce this effect.

Murine giardiasis has provided a useful model for the study of host parasite relationships in the intestine. For instance, the demonstration of prolonged infection in athymic, nude mice has indicated that immunological mechanisms are

*Dr Nair was supported by a British Commonwealth Medica 1 Fellowship. His present address is: Division of Gastroe rterolcry Medical College Hospital, Calicut-673008, Kerala, India.

†Address for correspondence and reprint requests: $\mathrm{Dr}$ Anne Ferguson, Gastro-Intestinal Unit, Western General Hospital, Crewe Road, Edinburgh EH4 2XU, Scotland.

Received for publication 12 December 1980 likely to be important in elimination of the parasite. ${ }^{34}$ We here report a short series of experiments in which the effects of corticosteroids on the course of mouse giardiasis infection have been examined. Also, perhaps of greater clinical relevance, we demonstrate that corticosteroid treatment leads to recrudescence of occult giardiasis in mice.

\section{Method}

A N I M A L S

Inbred, male CBA mice were used for all experiments, and for maintenance of a stock Giardia muris infection. Animals had free access to tap water and to a standard rodent diet (Stratt's mouse pellets) providing $\mathbf{4 \cdot 2}$ calories per gram, protein content $21 \cdot 3 \%$. Faecal specimens and intestinal contents of stock CBA mice were checked regularly to confirm the absence of protozoal and helminth infections in the colony.

G I A R D I A M U R I S I N F E C T I O N Giardia muris cysts were provided by $\mathrm{Dr}$ I Roberts-Thomson and flown from Australia to the United Kingdom by air mail. Stock mice were infected by oral inoculation of 1000 cysts, and the infection maintained by weekly infection of two or three adult CBA mice. 
For all experiments, male CBA mice aged 6 to 7 weeks were infected by intragastric administration of 1000 Giardia muris cysts in $0.2 \mathrm{ml}$ tap water. Cysts were isolated and counted by the method of Roberts-Thomson et $a l^{5}$ as described below

CORTISONE TREATM ENT

Cortisone acetate was injected subcutaneously in the interscapular region of mice. Control animals were given a subcutaneous injection of saline.

I S O L ATION OF CYSTS

Faeces from infected mice were broken up in tap water and the faecal suspension layered on molar sucrose of specific gravity $1 \cdot 11$, and centrifuged at $400 \mathrm{~g}$ for 15 minutes. Cysts, concentrated at the water/sucrose interface were removed, washed in normal saline, and resuspended in a known volume before counting in a haemocytometer.

TROPHOZOITE COUNT

A new technique was devised for enumeration of trophozoites in the small intestine The infected animal was killed by ether overdosage, the entire small intestine removed from the abdominal cavity, and the mesentery peeled off. Beginning with the duodenum, the intestine was everted over a spiral steel or glass rod, and the ends tied with black silk. The top part of the spiral rod was connected to a Chemap AG vibromix model tvne E1. which has a vibration frequency of $50 \mathrm{H}_{\mathrm{z}}$. The spiral was then placed in a dish containing $100 \mathrm{ml}$ of fluid $(98 \mathrm{ml}$ normal saline, $2 \mathrm{ml}$ acetyl cysteine, $20 \%$ Airbron, a mucolytic agent) at $4^{\circ} \mathrm{C}$ (Fig. 1). The intestine was then vibrated in medium for 10 minutes at the full power of the Vibromix. A $10 \mathrm{ml}$ sample of the medium was removed, centrifuged at $400 \mathrm{~g}$ for 10 minutes, the supernatant discarded, and the pellet resuspended in $0.5 \mathrm{ml}$ of medium, and trophozoites were then counted in a haemocytometer. The total trophozoite count per $100 \mathrm{ml}-$ that is, per intestine-was calculated. With this technique the limit of detection is 10000 trophozoites per animal.

\section{Fxperiments and results}

None of the infected or steroid treated animals showed ill effects during the experiments, none had diar thoea, and none died.

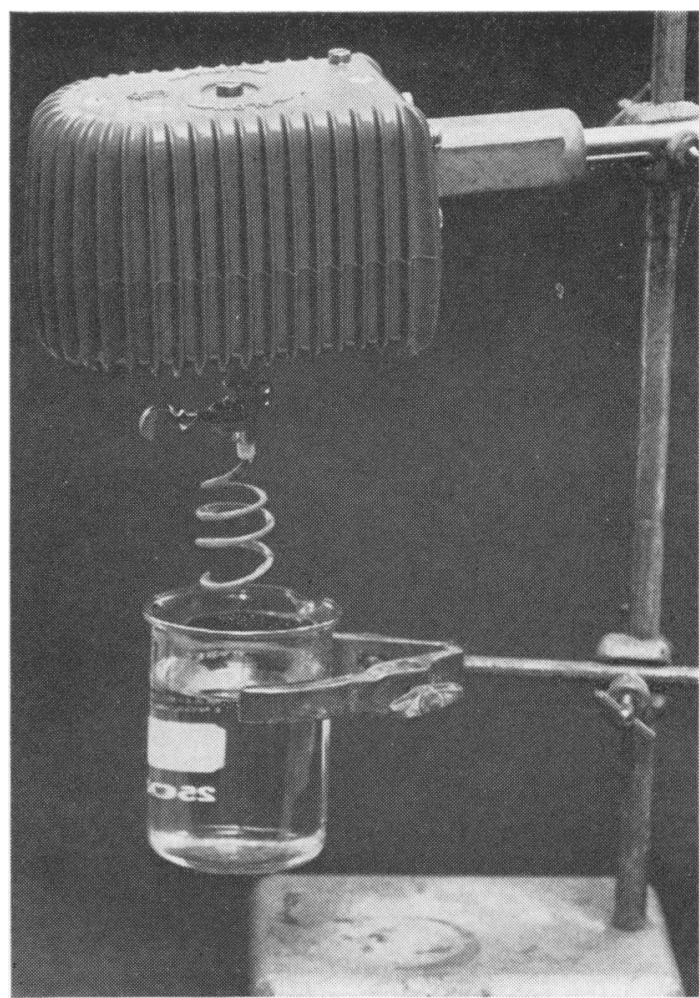

Fig. 1 Apparatus used for the 'vibration' method of counting Giardia trophozoites. The small intestine of a mouse is everted over the glass spiral as shown, and connected to a Chemap AG Vibromix.

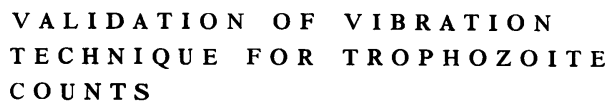

Preliminary experiments in animals which were heavily infected showed that sequential five to 10 minute periods of vibration of everted intestine, in changes of medium, resulted in recovery of between $97 \%$ and $100 \%$ of trophozoites within the first 10 minutes. After 10 minutes' vibration, no Giardia could be detected by histological examination or by examination of direct mucosal smears from the post-vibration specimen of intestine.

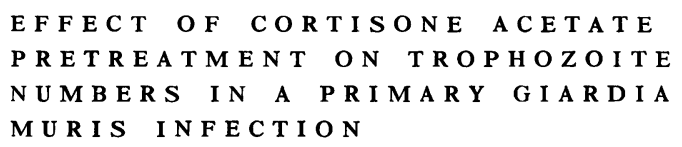

This experiment was designed to establish the effect of cortisone acetate, given one day before oral inoculation of cysts, on the time-course of primary infection and on the magnitude of the 
parasite load. Thirty-five male CBA mice were injected with $2.5 \mathrm{mg}$ cortisone acetate subcutaneously, and another 35, matched for age and sex, had $1 \mathrm{ml}$ saline subcutaneously. The following day all animals were infected with 1000 Giardia muris cysts. Five animals from each group were killed at one, two, three, four, six, eight, and 10 weeks post-infection. Trophozoite counts were carried out as described above. Results, shown in Fig. 2, illustrate that, in this experiment, trophozoite counts of saline treated animals reached a plateau between one and six weeks post-infection, with values of between 5 million and 10 million trophozoites per animal. In contrast, the trophozoite counts in cortisone pretreated animals were significantly higher than controls at one, two, three, and four weeks post-infection, values for individual animals at weeks 2 and 3 reaching as high as 25 million trophozoites. In both groups, there was a drop in trophozoite counts at eight and 10 weeks postinfection, and for the group of cortisone pretreated animals, the mean count at eight weeks was significan:ly higher than in the saline pretreated controls.

Thus, this experiment showed that cortisone acetate pretreatment modified the parasite load in primary Giardia infection, with substantially higher parasite numbers early in the course of infection (threefold increase at two weeks) and with a slower rate of drop in parasite numbers at two months post-infection.
In order to differentiate an effect of cortisone on the parasite from an effect on the specific and non-specific host immunity, animals were given cortisone acetate one and two weeks before Giardia infection and trophozoite counts were done in both groups of cortisone pretreated animals, and in saline injected controls, on the tenth day post-infection. Results, illustrated in Fig. 3, show that the parasite load was significantly higher than control in both cortisone acetate treated groups of animals. It is therefore likely that the effect of cortisone is exerted on the host, rather than by direct effect on the parasite.

\section{RECRUDESCENCE OF CHRONIC}

G I A R D I A I NFECTION

This aspect was studied in a group of 31 animals which had been infected with Giardia muris eight months previously. Nine of the animals were killed and trophozoite counts were done. Trophozoites were detected in only two of these nine mice. The remaining 22 animals were given $2.5 \mathrm{mg}$ cortisone acetate subcutaneously, and were killed in groups at one, two, three, five and 10 days after cortisone injection. Trophozoite counts were carried out. Results of this experiment are shown in Fig. 4. A few trophozoites were detected in one of the four animals killed on day one post-cortisone; thereafter, values of the order of five to 25 million tronhozoites were obtained for 10 of the animals and two others

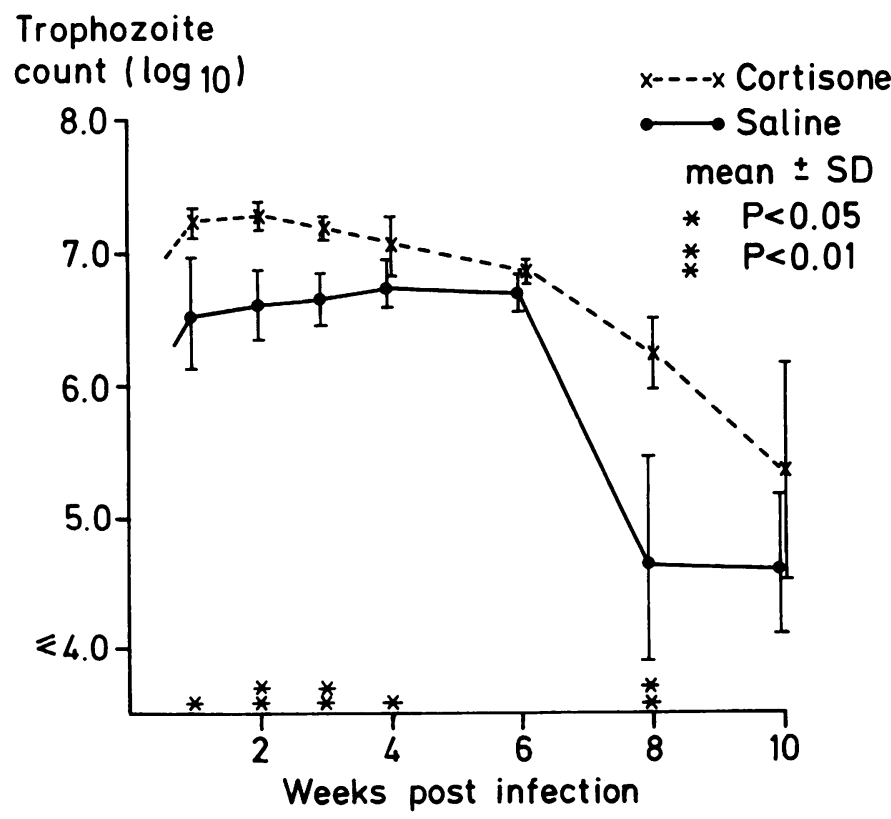

Fig. 2 Trophozoite counts (mean of five mice, $\pm S D$ ) at intervals after infection of $C B A$ mice by oral inoculation of 1000 Giardia muris cysts. $2.5 \mathrm{mg}$ cortisone acetate, or saline, was injected subcutaneously one day before infection. 
Trophozoite count $(\log 10)$

at 10 days post-infection

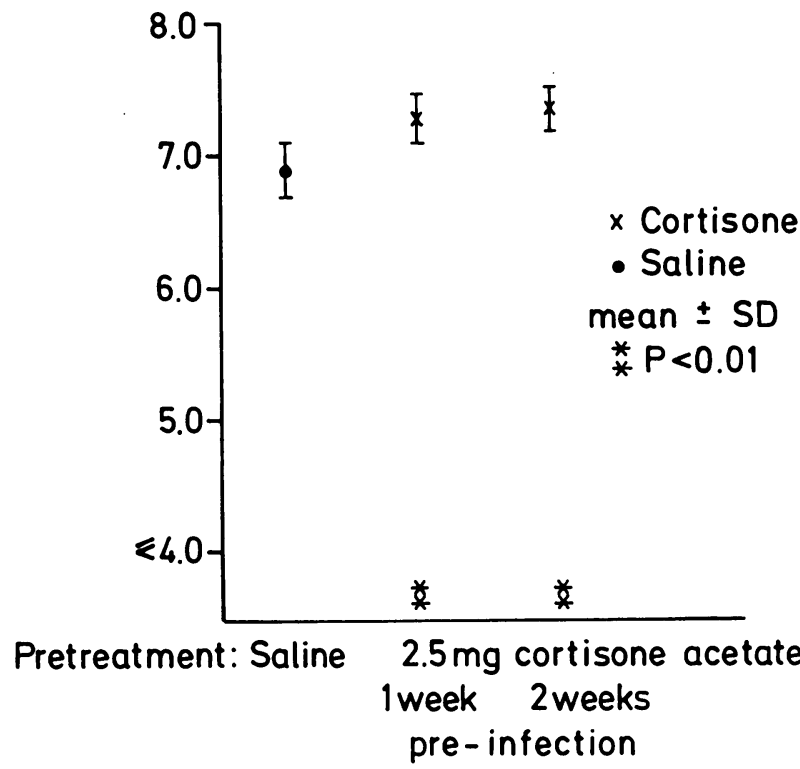

Fig. 3 Trophozoite counts (mean of five mice, $\pm S D)$ in animals treated with saline, or with $2.5 \mathrm{mg}$ cortisone acetate subcutaneously, one or two weeks before oral inoculation of 1000 Giardia muris cysts.

had lower trophozoite counts. Trophozoites were not detected in five.

It seems likely that $25 \%$ of animals have completely cleared the parasite by eight months after primary infection. Another $50 \%$ have small numbers of trophozoites, below the limit of detection of this assay system, which was 10000 trophozoites per animal; but after a single injection of cortisone acetate the trophozoite numbers increase very rapidly, to reach values analagous to those in a primary infection, by the second day after cortisone administration.

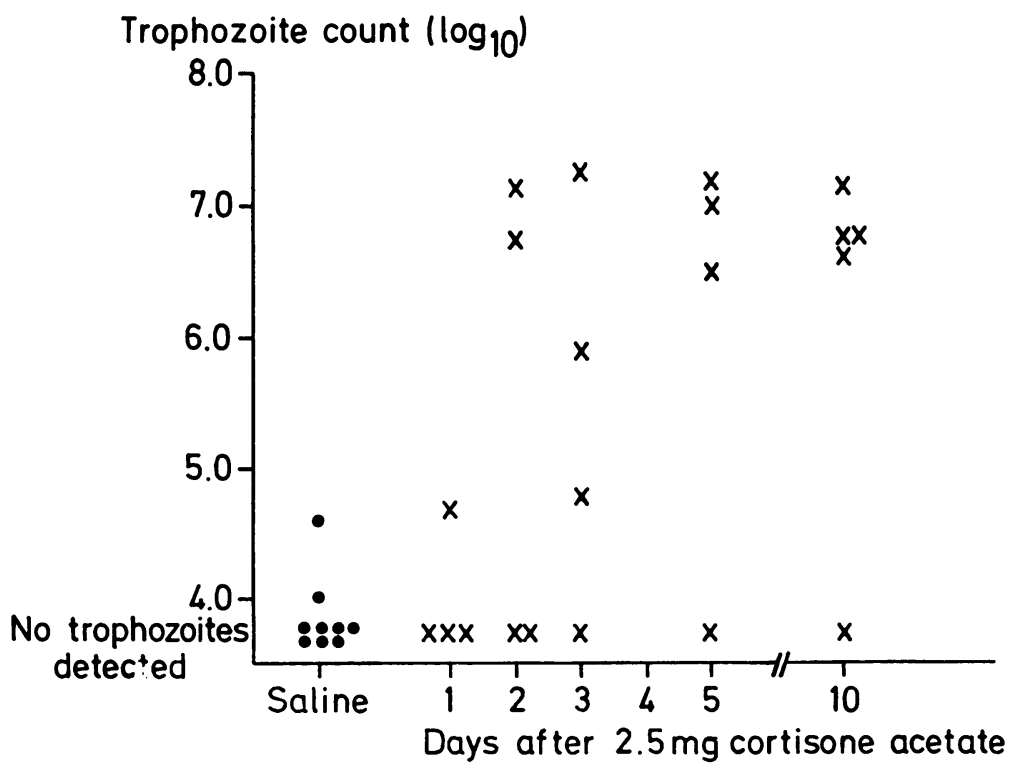

Fig. 4 Trophozoite counts in mice, eight months after oral inoculation of 1000 Giardia muris cysts, and one to 10 days after $2.5 \mathrm{mg}$ cortisone acetate or saline was injected subcutaneously. Limit of detection of trophozoites was 10000 cysts per animal. 


\section{Discussion}

The parasite-related and host-related facets of the changing host parasite relationship in giardiasis can readily be examined in experimental animals. The course of infection can be monitored indirectly by counting faecal cyst excretion. However, as many factors influence the rate of cyst formation and excretion, direct counts of trophozoites, as in the present study, are preferable. In these experiments, as in previously reported studies of normal ('BA mice," " oral inoculation of 1000 Giardia muris cysts led to a predictable infection, with five to 10 million trophozoites in the small intestine from the first week after infection. There was a decline in the number of parasites in the second month, and thereafter most animals have been found to continue to have low grade infection, with small numbers of trophozoites present in the intestine, and intermittent excretion of cysts (submitted for publication). The mechanism by which the number of trophozoites is controlled is still unknown. Giardia trophozoites divide by binary fission, every five hours or so, ${ }^{7}$ so the trophozoite load can increase very rapidly. Some parasites will be lost by faecal excretion, usually as cysts, and it is likely, but not proven, that parasites can be killed or excreted as a result of thymus-dependent immunological reactions. Infection is prolonged and persistent in athymic nude mice ${ }^{3} 4$ and a reduction in parasite numbers can be obtained by transfer of immune cells between animals. ${ }^{3}$ However, thymus-independent factors are likely also to play a role, for even in athymic mice the number of parasites eventually decreases slowly, and it has been proposed that macrophages play a crucial role in non-specific anti-Giardia immunity. ${ }^{8}$

In the series of experiments reported in this paper, a single injection of a slow release corticosteroid, cortisone acetate, has been used to examine the effects of non-specific immunosuppression on the host parasite relationship in giardiasis. We reasoned that immunosuppression could influence the pattern of a primary infection by producing changes in the maximum parasite load attained, in the duration of the period of heavy infection, and might alter the rate at which the number of parasites drops during the second month. An attempt to produce recrudescence of occult infection was included in the protocol when other experiments showed the persistence of a very small number of trophozoites many months after primary in- fection, in the strain of animals used. These experiments have now shown unequivocally that cortisone acetate injection produces significant alterations in the course of a primary infection. The number of parasites within the small intestine was significantly higher than in saline injected controls for four weeks after infection. The drop in number of parasites after week six was also slower in the cortisone pretreated animals. The effects could be reproduced by injection of cortisone up to two weeks before infection by oral administration of the cysts, so that effects on the host's specific or non-specific immunity, rather than a direct trophic effect of cortisone on the parasite, is the likely mechanism. We also observed that a single dose of cortisone acetate induced recrudescence of occult Giardia infection in mice within 48 hours of its administration, and this, indirectly, confirmed that some $75 \%$ of animals still had a few parasites within the intestine even eight months after infection.

Corticosteroids influence various facets of immunity. They alter antigen processing by macrophages, ${ }^{910}$ produce atrophy of the thymus and dramatic lympholysis ${ }^{11} 12$ and high doses of cortisone acetate, as used in the present study, also significantly reduced the concentrations of serum immunoglobulins in mice. ${ }^{13}$ The mechanism whereby steroids act in this infection has not been elucidated by these experiments, and we recognise that, in addition to specific effects on the immune system, steroids may also have blocked or suppressed the effects of inflamatory mediators, and that non-immunological factors, such as the intestinal microbial flora, and the structure and properties of enterocytes, should also be considered.

The observations in this study may be of clinical relevance. Many parasites persist in the gastrointestinal tract of the host for months or years, although the infection remains clinically inapparent. Immunosuppressive therapy in such individuals has been shown to produce recrudescence of occult infections, in the case of amoebiasis $^{1}$ and strongyloidias. ${ }^{14}{ }^{15}$

Giardiasis is a cosmopolitan infection, and under insanitary and tropical conditions the prevalence may reach $20 \%$ with higher rates in children than in adults. In such areas, intestinal parasite infection is a factor to be considered when an immunosuppressed patient develops diarrhoea. We now propose to undertake clinical studies to examine the significance of occult giardiasis in such patients, and to 
establish whether recrudescence of parasite infections in immunosuppressed individuals produces asymptomatic infection or, more likely, a clinical illness with malabsorption similar to that found in patients with hypogammaglobulineamia. ${ }^{17}$

We acknowledge the excellent technical help of Mr A Sutherland, and of the staff of the Animal Unit, Western General Hospital.

\section{References}

1Stuiver PC, Goud TJLM. Corticosteroids and liver amoebiasis. $\mathrm{Br}$ Med J 1978; 2:394-5.

${ }^{2}$ Abioye AA. Fatal amoebic colitis in pregnancy and puerperium. J Trop Med Hyg 1973; 76:97-100.

${ }^{3}$ Roberts-Thomson IC, Mitchell GF. Giardiasis in mice. I Prolonged infection in certain mouse strains and in hypothymic mice. Gastroenterology 1978; 75:42-6.

${ }^{4}$ Stevens DP, Frank DM, Mahmoud AAF. Thymus dependency of host resistance to Giardia muris infection: studies in nude mice. J Immunol 1978; 120:680-2

${ }^{5}$ Roberts-Thomson IC, Stevens DP, Mahmoud AAF, Warren KS. Giardiasis in the mouse: an animal model. Gastroenterology 1976; 71:57-61.

${ }^{6}$ Ferguson A, Gillon J, Al Thamery D. Intestinal abnormalities in murine giardiasis. Trans $R$ Soc Trop Med Hyg 1980; 74:445-8.

${ }^{7}$ de Carneri I, Trane F, Mandelli V. Giardia muris: oral infection with one trophozoite and generation time in mice. Trans $R$ Soc Trop Med Hyg 1977; 71:438.
${ }^{8}$ Owen RL, Allen L, Stevens DP. Intraepithelial trapping of Giardia by macrophages in Peyer's patches of normal and nude mice-an intestinal defense and antigen processing mechanism (Abstract). Gastroenterology 1980; 78:1232.

${ }^{9}$ Thomas L. Cortisone and infection. Ann NY Acad Sci 1953; 56:799-813.

${ }^{10}$ van Dijk H, Slotboom A, van Bohemen CG, Willers JM. Immunomodulation by macrophages. In: Werner GH, Floc'h F, eds. Pharmacology of Immunoregulation. New York: Academic Press, 1978; 207-15.

${ }_{11}$ Makman MH, Nakagawa S, White A. Studies on the mode of action of adrenal steroids on lymphocytes. In: Pincus G, ed. Recent progress in hormone research 1967; 23:195-227.

${ }^{12}$ Schechter B, Feldman M. Hydrocortisone affects tumour growth by eliminating precursors of suppressor cells. J Immunol 1977; 119:1563-8.

${ }^{13}$ Levy AL, Waldmann TA. The effect of hydrocortisone on immunoglobulin metabolism. J Clin Invest 1970; 49:1679-84.

${ }^{14}$ Willis AJP, Nwokolo C. Steroid therapy and strongyloidiasis. Lancet 1966; 1:1396-8.

${ }^{15}$ Neefe LI, Pinilla O, Garagusi VP, Bauer H. Disseminated strongyloidiasis with cerebral involvement, a complication of corticosteroid therapy. $\mathrm{Am}$ J Med 1973; 55:832-7.

${ }_{16}^{16}$ Petersen H. Giardiasis (lambliasis). Scand $J$ Gastroenterol 1972; 7(suppl 14):1-44.

${ }^{17}$ Ament ME, Ochs HD, Davis SO. Structure and function of the gastrointestinal tract in primary immunodeficiency syndromes. Medicine 1973; 52: $227-48$. 